\title{
NOTE
}

\section{Measurements of Evaporation Rates of Water}

Mass transfer rates were measured during evaporation of water into its own vapor both from a stagnant pool and from a flowing surface which was replenished continuously. The water surface temperature was determined by comparing the intensity of the infrared radiation emitted by the surface with that emitted by a black cavity of known temperature. In addition to the good accuracy afforded by this technique, it also offers the advantages that there is no physical contact between the surface and the instrument, and that the recorded temperature is averaged over only a very thin liquid layer.

The experimental apparatus is shown in Fig. 1. Evaporation took place from a reservoir located in the center of the vacuum chamber. Two different types of reservoirs were used (Fig. 2). The first type was a cylindrical container containing a stagnant pool of water. Two interchangeable containers (with $7 \mathrm{~cm}$ and $5 \mathrm{~cm}$ id) were used to achieve a reasonably wide range of mass transfer rates. The second type of reservoir consisted of a small "upper" reservoir, a main reservoir, and a collector. Water, fed by drops into the upper reservoir, flowed at a continuous rate into the main reservoir through an overflow. The water from the main reservoir was passed into a sealed collector tank. The water temperature was regulated by thermofoil heaters (surrounded by teflon insulation) attached to the bottoms of the reservoirs. Triple distilled water was used in the experiments and was outgassed before it was admitted into the reservoir.

In the experiments using the cylindrical water containers the evaporation rate was obtained by measuring the change in the height of the water level during a given period of time $(\sim 5 \mathrm{~min})$. The level of the surface was determined by observing the position of the tip of a needle when it contacted the surface. The mass transfer rate was also established as a function of the vapor pressure in the vacuum chamber and the opening of the valves leading to the pump. This information was used in determining the evaporation rate when the water level could not be measured directly. In the case of the flowing system, mass transfer from the overflow was estimated to be less than $\sim 1 \%$ of the total mass transfer rate.

The radiation emitted by the water surface was compared to the radiation emitted by a black body maintained at a known temperature. The black body, mounted on top of the vacuum system, consisted of a $16.5 \mathrm{~cm}$ high hollow aluminum cone (wall thickness $0.2 \mathrm{~cm}$ ) with a bottom area of $12.5 \mathrm{~cm}^{2}$. Special coating material was sprayed on the inside surface to increase the emissivity. The temperature of the cone was controlled by constant temperature air blown over it, and was measured by two copper-constantan thermocouples embedded in the wall.

Radiation from the black body and from the water surface was transmitted to the infrared detector through an optical system consisting of a stationary reflector, a chopper, an infrared-transmitting window, and a concave mirror. The stationary reflector was a first surface mirror, its purpose being to reflect the infrared rays emitted by the black body toward the chopper. The $10 \mathrm{~cm}$ diam chopper resembled a "clover leaf." The four "leaves" were covered with first surface mirrors. As the chopper rotated it alternately (at $20 \mathrm{cps}$ ) 


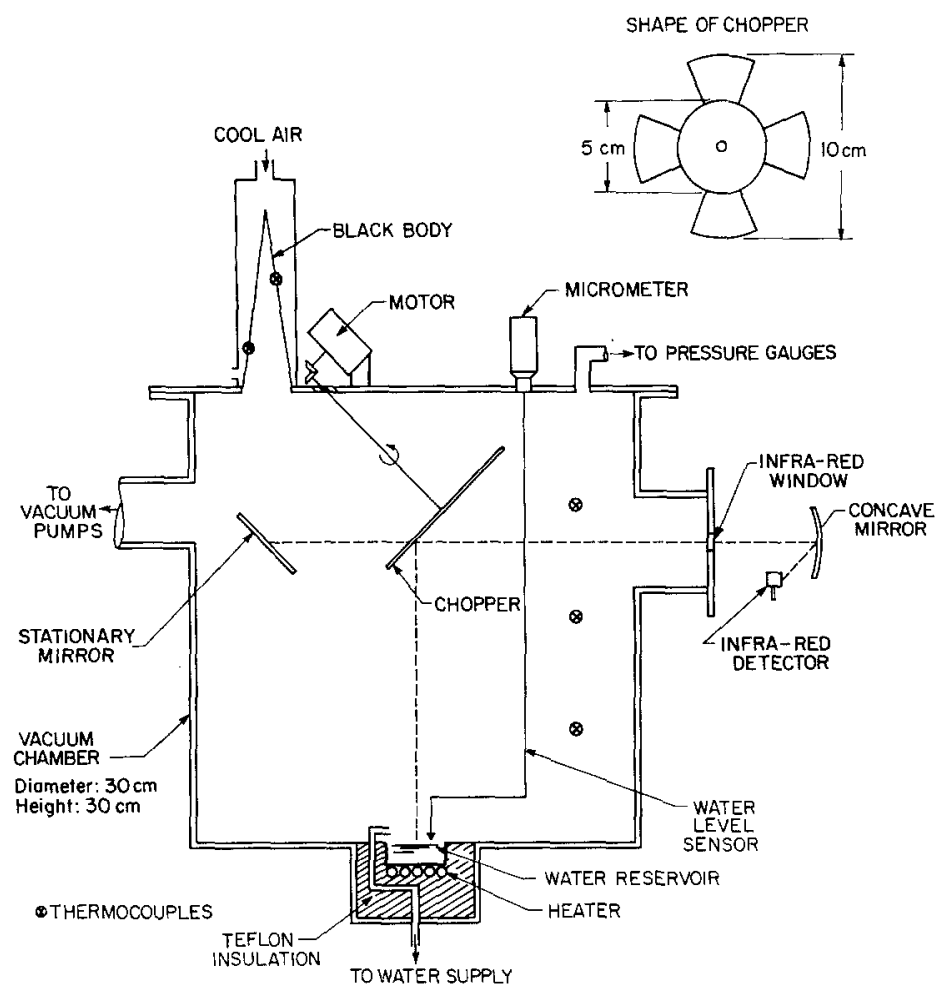

FIG. 1. Schematic of apparatus.

reflected towards the detector the radiation emitted either by the black body or by the water surface. The black body and the water surface were at equal distances from the chopper.

The window $(0.3 \mathrm{~cm}$ thick, $2.5 \mathrm{~cm}$ diam) was made of IRTRAN, and had a uniform transmittance of about $70 \%$ for wavelengths within 1-14 $\mu \mathrm{m}$. In this range the emissivity of water is nearly unity and is nearly independent of wavelength (1). The infrared detector was placed at the focal point of a concave mirror mounted outside the window.

The infrared detector was a bolometer with a detector area of $0.0625 \mathrm{~cm}^{2}$, time-constant of $2.5 \mathrm{msec}$, and a response of $20 \mathrm{~V} / \mathrm{W}$. The

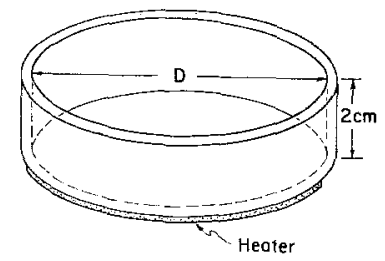

$D=5$ or $7 \mathrm{~cm}$

(a)

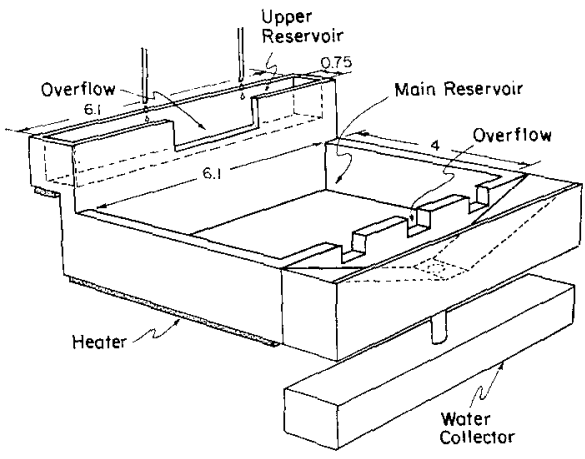

(b)

FIG. 2. Schematic of water reservoirs. (a) Reservoir used for stagnant surface, (b) reservoir used for flowing water (all dimensions in $\mathrm{cm}$ ). 


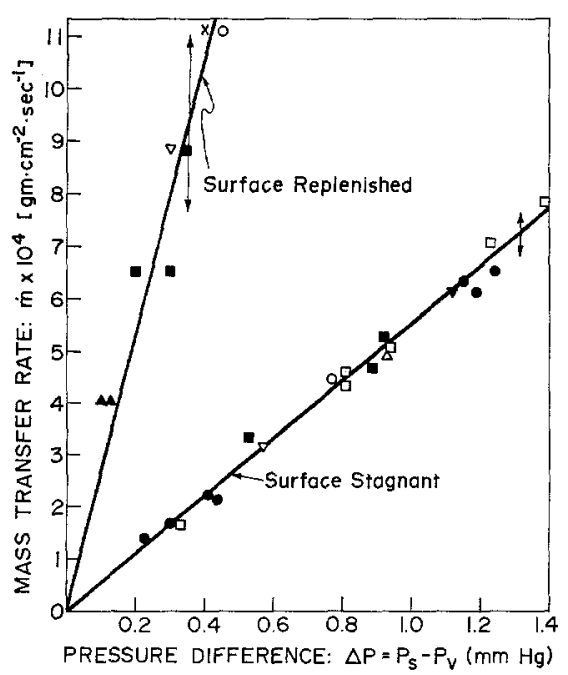

FIG. 3. Mass transfer rate versus pressure difference during evaporation. Data $\otimes T_{s}=18-19^{\circ} \mathrm{C} ; 0$ $T_{s}=19-20^{\circ} \mathrm{C} ; \bullet T_{s}=20-21^{\circ} \mathrm{C} ; \square T_{s}=21-22^{\circ} \mathrm{C}$; $T_{s}=22-23^{\circ} \mathrm{C} ; \nabla T_{s}=23-24^{\circ} \mathrm{C} ; \boldsymbol{\Delta} T_{s}=24-25^{\circ} \mathrm{C}$; $\nabla T_{s}=25-26^{\circ} \mathrm{C} ; \triangle T_{s}=26-27^{\circ} \mathrm{C}$. Solid line: Least square fit to data. Arrows indicate magnitude of error.

electrical signals from the bolometer (generated by the infrared radiation received alternately from the black body and the water surface) were transmitted to a signal-tuned amplifier through a preamplifier. The signal tuned amplifier was used to amplify the signal and to filter out most of the noise. A reference signal is required for this amplifier; it was generated by a collimated beam of light shining on a phototransistor. The light beam was interrupted by a chopper at 20 cps. A meter contained in the unit indicated the root mean square value of the difference in intensity between the radiation from the black body and from the water surface.

The surface temperature, measured in the manner just described, corresponds to the average temperature of a thin water layer only. The thickness of this layer was found by calculating the $e$-folding depth for absorption of the incident radiation at a given wavelength, and by averaging the results over the 1-14 $\mu \mathrm{m}$ wavelength range detected in the experiments. These calculations resulted in a thickness of $0.001 \mathrm{~cm}$ (2).
The entire surface areas of both circular reservoirs and of the main (large) rectangular reservoir were scanned with the bolometer. No measureable differences could be detected in the surface temperature across any of these surfaces. The surface temperature of the small upper (rectangular) reservoir could not be measured accurately because of the slight ripples caused by the dripping water. Measurements indicated that the temperature of this surface was below the temperature of the main reservoir by at most $0.5^{\circ} \mathrm{C}$. The maximum error caused by this temperature difference was estimated to be about $10 \%$.

The pressure in the vacuum chamber was measured with an ionization gauge, a McLeod gauge and a $U$-tube mercury manometer. The pressure distribution in the vapor above the liquid surface was also measured with an inclined manometer. The vapor temperature was measured with copper-constantan thermocouples.

\section{RESULTS AND DISCUSSION}

Evaporation rates of water were measured at several different pressure differences $\Delta P$ $=P_{s}-P_{v}$, where $P_{s}$ is the saturation pressure corresponding to the water surface temperature $T_{s}$, and $P_{v}$ is the vapor pressure. The highest $\Delta P$ in the experiments was $\sim 1.4$ $\mathrm{mm} \mathrm{Hg}$. At higher pressure differences it was difficult to avoid the formation of bubbles in the reservoir. The surface temperature ranged from 18 to $27^{\circ} \mathrm{C}$. The results are shown in Fig. 3. The lower line represents data obtained during evaporation from a stationary pool of water. The upper line shows the evaporation rate from the surface of flowing water. When the surface was constantly replenished the evaporation rate increased by a factor of five, indicating the serious effect of surface contamination on the interfacial mass transfer.

Above $0.1 \mathrm{~cm}$ from the surface the vapor pressure varied only by a maximum of $0.5 \%$, and above $2.5 \mathrm{~cm}$ it did not change appreciably. This suggests that the results of the one dimensional analyses of Schrage (3) and Patton 
and Springer (4) may be used to calculate the condensation and evaporation coefficients, $\sigma_{e}$ and $\sigma_{e}$. For the conditions of the present experiments $\left(T_{\mathrm{s}}-T_{v} / 2 T_{v} \cong 0.003, \Delta P / P_{v}\right.$ $>0.015)$ the mass transfer rate from the surface may be expressed as $(3,4)$

$$
\begin{aligned}
\dot{m} \cong \rho_{\nu}\left(\frac{R T_{v}}{2 \pi}\right)^{\frac{2}{2}} \frac{1}{\left(1 / \sigma_{c}\right)+B} & \\
& \times\left(\frac{\sigma_{e}}{\sigma_{c}} \frac{\Delta P}{P}+\frac{\sigma_{e}}{\sigma_{c}}-1\right),
\end{aligned}
$$

where $R$ is the gas constant, $\rho_{v}, T_{v}$ the density and temperature of the vapor far away from the surface. $B$ is a constant having values of either -0.5 (Schrage) or 0.125 (Patton and Springer).

It is noted from Fig. 3 that $\dot{m}$ varies linearly with $\Delta P$ and the curve passes through the origin (equilibrium point $\dot{m}=0, \Delta P=0$ ). According to Eq. [1], under these circumstances $\sigma_{c}$ and $\sigma_{e}$ are constant and are equal, i.e., for the conditions of the present experiments $\sigma_{c}=\sigma_{e}=\sigma$. A similar conclusion is reached when Eq. [1] is applied to Hammecke and Kappler's data (5), obtained for water near equilibrium. This equality was also found byWylie and Brodkey (6), who measured the condensation and evaporation coefficients of mercury.

We calculated $\sigma$ from Eq. [1] by setting $\sigma_{c}=\sigma_{e}=\sigma$ and using the experimentally measured values for $\dot{m}, \Delta P, P_{v}$, and $T_{v}$. The results are

$$
\begin{array}{rr}
\sigma_{\text {Schrage }}=0.038, & \sigma_{\text {Patton-Springex }}=0.038 \\
& (\text { surface stagnant }), \\
\sigma_{\text {Schrage }}=0.17, & \sigma_{\text {Patton-Springer }}=0.19 \\
\text { (surface replenished) } .
\end{array}
$$

The uncertainties in $\sigma$ caused by the measurements are about two percent. The above results show the effect of surface contamination on $\sigma$. For a stagnant surface the $\sigma$ values are five times lower than for a flowing (and presumably less contaminated) surface. In the latter case the surface still may contain contamination of course, and the actual value of $\sigma$ for a perfectly clean surface may possibly be higher than the values given above. The present lower values are similar to those found in previous experiments on evaporation from a stationary flat surface or from a spherical droplet. The higher $\sigma$ values here obtained are of the same order of magnitude as those measured in filmwise condensation or waterjet experiments, in which the water surface was constantly being changed.

\section{ACKNOWLEDGMENTS}

This work was supported by the U. S. Atomic Energy Commission and by the National Science Foundation.

\section{REFERENCES}

1. Wolfe, W. L., "Handbook of Military Infra-Red Technology," Office of Naval Research, Department of the Navy, Washington, DC, 1965.

2. Narusawa, U., Ph.D. Thesis, University of Michigan, 1971.

3. Schrage, R. W., "Interphase Mass Transfer," Columbia University Press, New York, 1953.

4. Patton, A. J., and Springer, G. S., in "Rarefied Gas Dynamics" (L. Trilling and H. Y. Wachman, Eds.), Vol. 2, p. 1497. Academic Press, New York, 1969.

5. Hammecke, V. K., and Kappler, E., Z. Geophys., 19, 181 (1953).

6. Wylie, K. F., AND Brodkey, R. S., International Symposium on Two-Phase Systems, Technion City, Haifa, Israel, Paper No. 1-1, 1971.

Utchiro Narusawa GEORGE S. SPRINGER

Fluid Dynamics Laboratory,

Department of Mechanical Engineering,

The University of Michigan,

Ann Arbor, Michigan 48104

Received July 1, 1974; accepled A ugust 22, 1974 